\title{
OPEN SOURCE
}

\section{Unentdeckte Potenziale}

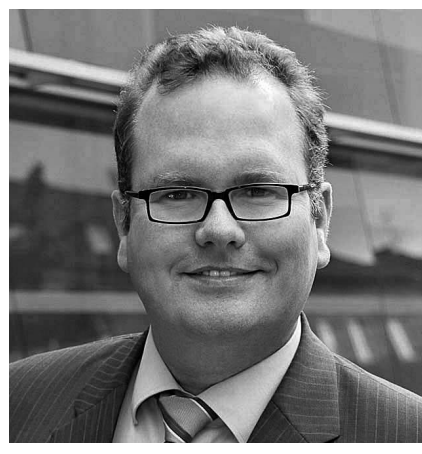

VON PETER FAISS

Der Diplom-Betriebswirt (BA)

Peter Faiß ist Inhaber der Imendo

Unternehmensberatung. Er ist

seit mehr als zwanzig Jahren als Unternehmensberater tätig und hat Träger der Sozialwirtschaft zum IT- und Verwaltungsmanagement beraten.

Faiss@Imendo.de

\author{
Open-Source-Software spielt in der Sozialwirtschaft \\ bislang keine große Rolle. Doch spätestens mit \\ den deutlichen Preiserhöhungen von Microsoft \\ für die Sozialwirtschaft rücken die freien \\ Programme in das Blickfeld der Entscheider.
}

Unter Open Source Software (OSS) werden Programme verstanden, die ehrenamtlich von freien Entwicklergemeinschaften als alternativer Entwurf zur kommerziellen Industrie der Informationstechnologie (IT) entwickelt wird. Der Quellcode von Open Source ist im Gegensatz zu herstellergebundener (»proprietärer«) Software öffentlich zugänglich, prüf- und veränderbar und kann je nach Lizenzmodell geändert, weitergegeben und in eigenen Produkten weiterverwendet werden.

Bekannte Open Source-Systeme sind das Betriebssystem Linux, der Webbrowser Firefox, das Mailprogramm Thunderbird, die Office-Systeme LibreOffice und Apache OpenOffice sowie Web-site-Software wie Wordpress, Typo3 oder Joomla.

Die Open Source Initiative (OSI) ist eine gemeinnützige Organisation, die mit ihrer Open Source Definition einen weltweit akzeptierten Standard gesetzt hat (http://opensource.org/docs/osd). In der Open Source Definition sind Anforderungen an Open Source Software wie freie Weitergabe, verfügbarer Quellcode, freie Weitergabe weiterentwickelter Arbeiten, keine Diskriminierungen von Personen oder Gruppen, keine Nutzungseinschränkung, Produktneutralität etc. formuliert.

Während Shareware nur einen begrenzten kostenlosen Testzeitraum gewährt, kann Open-Source-Software unbegrenzt kostenlos eingesetzt. Shareware wird meist - im Gegensatz zu Open-Source-Software - nicht in einer Entwicklergemeinschaft, sondern von einzelnen Entwicklern und kleinen Unternehmen entwickelt, die Quellcodes sind bei Shareware nicht öffentlich. Freeware bezeichnet Software, die als ausführbares Programm zur kostenlosen Nutzung verbreitet werden, deren Quellcodes ebenfalls nicht veröffentlicht werden.

Grundlegende Open-Source-Gedanken liegen auch inhaltlichen Angeboten wie beispielsweise Wikipedia, die freie Enzyklopädie im Internet, zugrunde. Doch nicht jeder gemeinschaftlich erarbeitete Inhalt im Internet basiert auf diesem Gedankengut: Viele Unternehmen öffnen ihre Webauftritte für Produkt- oder Dienstleistungsbewertungen, die anwenderseitig (Crowd) erstellt werden. Diese Bewertungen werden in kommerziellem Interesse eingeholt und gegebenenfalls sogar im Anbieterinteresse manipuliert (z. B. Bewertungen in Hotel- und Reiseportalen).

Im Sinne von Open Source Software betrieb der Verein zur Förderung freier Informationen für die Pflege e. V. das deutschsprachliche »PflegeWiki« (www. pflegewiki.de). Es war eine Plattform zur gemeinschaftlichen Bereitstellung pflegerelevanter Inhalte. Der Betrieb wurde allerdings im Herbst 2012 eingestellt.

Für die Informationstechnologie sind neben der eigentlichen Open-SourceSoftware auch die in diesem Zusammenhang entstehenden Standards für die Datenablage und den Datenaustausch attraktive Gestaltungsfelder. Die Marktmacht kommerzieller Softwareanbieter kann auch über die eingesetzten Datenformate gestaltet und bewahrt werden. 
Der Open-Source-Bewegung ist es zuzurechnen, dass in vielen Fällen proprietäre Datenformate geöffnet wurde und freie Standards berücksichtigt wurden.

Als eher klassisch-propritären Ansatz kann das vom Bundesverband evangelische Behindertenhilfe e. V. (www.bebev.de/) propagierte Verfahren GBM (EDV-gestütztes Verfahren zur Qualitätssicherung und Qualitätsgestaltung in sozialen Einrichtungen, www.gbm. info) verstanden werden. Das Verfahren ist an einen Softwareanbieter gebunden und konnte sich in der Behindertenhilfe bislang nicht flächendeckend durchsetzen.

Die meisten Open-Source-Angebote setzen branchenunabhängige Anwendungen um: Betriebssysteme, IT-Servicemanagement, Webbrowser, OfficeAnwendungen, Content Management Systeme etc. Durch die Branchenunabhängigkeit lassen sich mit diesen Anwendungen große Anwender- und Entwicklergruppen erreichen. Im klassischen Internet-Umfeld haben diese branchenunabhängigen Open Source Software-Anwendungen auch in der Sozialwirtschaft eine hohe Anwendung und Akzeptanz gefunden.

Eine deutlich geringere Akzeptanz konnte das Open-Source-Betriebssystem Linux in der Sozialwirtschaft finden. Nahezu alle Fachanwendungen der Sozialwirtschaft setzen die MicrosoftProdukte als technische Basis voraus. Linux konnte in der Sozialwirtschaft im Betrieb von Webanwendungen (Mailserver, Homepages etc.) sowie von Infrastructure-as-a-Service-Angeboten (IaaS) in der Cloud eine Nische besetzen. Während die IaaS-Angebote für den Betrieb von Open-Source-Software weiterhin meist auf Linux aufsetzen, werden nun zunehmend IaaS-Angebote mit den Microsoft-Betriebssystemen angeboten. Die MS-Lizenzen sind bei diesen Angeboten in den nutzungsbasierten Preisen bereits enthalten.

Im Bereich der Office-Anwendungen haben Produkte von Open Source Software wie LibreOffice und Apache OpenOffice bislang eine beschränkte Verbreitung gefunden: Listen und Auswertungen können in vielen Fachsystemen zu den Open Source Software-Produkten exportiert werden. Mit steigendem Integrationsgrad - Rücklauf aus dem Office-System in die Fachanwendung, integrierte Controlling-Cockpits etc.
- nimmt die Unterstützung von Open Source Software der branchenspezifischen Fachsysteme stark ab.

Die Bindung an die Microsoft-Standards umfasst in den Fachsystemen ein breites Spektrum bis zur vollständigen technischen Integration sowie der kompletten Übernahme der Benutzeroberflächenstandards. Microsoft hat mit Office 2007 die Menüführung über Menübänder (»Ribbons«) eingeführt. Während die einheitliche Ribbon-Gestaltung von Office und Fachsystemen bei vielen Anwendern geschätzt wird, findet der Gegenentwurf von klassischer Benutzeroberfläche im Open-Source-SoftwareOffice mit einer entsprechend klassischen Fachsoftware ebenso vehemente Verfechter.

Für die branchenspezifischen Anwendungen der Sozialwirtschaft (Klientenverwaltung, Pflege- und Hilfemanagement, Dienstplanung) hat sich bislang kein nennenswertes Angebot an Open Source Software oder eine entsprechende Entwicklergemeinschaft gebildet. Und dies, obwohl die Sozialwirtschaft ja über umfassende Erfahrungen mit ehrenamtlichem Engagement verfügt. Hier scheint die (noch) geringe IT-Affinität vieler in der Sozialwirtschaft Tätiger ein prägender Einfluss zu sein. Zudem führt die Komplexität der Fachsysteme in ihrer branchenspezifischen Ausprägung zu einem ungünstigen Verhältnis von Entwicklungsaufwand zu potenzieller Entwicklergemeinschaft.

Open-Source-Software ist also für Kernanwendungen der Sozialwirtschaft (noch) kein Thema. Im Zusammenhang mit den schnell wachsenden und kostengünstigen Angeboten von Cloud-Lösungen werden auf die IT-Abteilungen der Sozialwirtschaft neue Herausforderungen zukommen: Bevor die IT-Abteilung das Wort »Datenschutz « aussprechen kann, haben IT-affine Mitarbeiter einen Server mit einem Open Source Software-Kontaktmanagement-System (Customer Relationship Management wie z. B. Sugar-CRM) beim Cloudprovider "hochgezogen " und in Betrieb genommen! Und das zu geringen Mietkosten und ohne Vertragsbindung über die Nutzungs- oder Testzeit hinaus.

\section{Rechtliches}

Im Wettbewerb mit herstellergebundener Software wurde verbreiteter Open-
Source-Software Rechteverletzungen von kommerziellen Anbietern vorgeworfen. Intensiv wurden Rechteverletzungen im Umfeld des Betriebssystems Linux durch die SCO Group verfolgt, auch gegenüber Anwenderunternehmen. Die SCO Group ist mit ihrem Ansinnen gescheitert, die juristischen Auseinandersetzungen haben sich auf die zahlreichen Patente im Telekommunikationsmarkt verlagert.

Für einen rechtssicheren IT-Betrieb sind die folgenden Maßnahmen zu empfehlen:

- Dokumentation der eingesetzten ITAnwendungssysteme

- IT-Nutzungsrichtlinie mit Regelungen zum Softwareeinsatz und zur Weitergabe von Software (z. B. an Ehrenamtliche)

- Prüfung der Rechtsrisiken, Abgleich mit Standardlizenztypen etc.

Die wichtigsten Lizenzformen von Open Source Software sind GPL (General Public License) und BSD (Berkeley Software Distribution).

\section{Arbeitskreis Open Source}

Der Fachverband Informationstechnologie in Sozialwirtschaft und Sozialverwaltung (FINSOZ e. V.) hat im Herbst 2012 einen Arbeitskreis »Open Source Software« ins Leben gerufen. Ausgelöst durch ein Fachforum zur Perspektive der Office-Anwendungen in der Sozialwirtschaft, wurde das Interesse an Open Source Software in der Sozialwirtschaft deutlich. Der Arbeitskreis geht mit den folgenden Zielen an den Start:

- Transparenz Open Source SoftwareAngebote für die Sozialwirtschaft

- Förderung und Etablierung offener Standards in der Sozialwirtschaft

- Plattform für Open Source SoftwareInitiativen, SW-Anbieter im Dialog mit Leistungsanbietern und Leistungsträgern

- Plattform für den Erfahrungsaustausch Open Source Software

Informationen, Ansprechpartner und Aktivitäten zum Arbeitskreis »Open Source Software« des FINSOZ finden sich auf der Verbandswebsite (www. finsoz.de). 


\section{Fazit}

Das Gedankengut von Open Source fördert als Gegenpol zu kommerziellen Lösungen die Entwicklung freier ITAnwendungssysteme, freier Inhalte und freier Standards. Die Sozialwirtschaft setzt bereits heute Open-Source-Software in den branchenneutralen Anwendungsbereichen wie Webanwendungen, Mail, Zusammenarbeit und in Ansätzen bereits Office ein.

Obwohl die Sozialwirtschaft im Ehrenamt umfassende Kernkompetenzen besitzt, hat sich für die branchenspezifischen Anwendungen in der Sozialwirtschaft noch keine Entwicklungsgemeinschaft gebildet. Der FINSOZ e. V. wird das Thema Open Source in der Sozialwirtschaft aufnehmen und fördern. Open Source ist für die Sozialwirtschaft einen Blick wert!

\section{Häufe Fragen zu Open Source in der Sozialwirtschaft}

Ist Open Source Software kostenlos?

$J a$, für Open-Source-Software fallen keine Lizenz- und Updatekosten an. Häufig sind jedoch kostenpflichtige Dienstleistungen rund um die Installation oder den Betrieb erforderlich.

Kann ich mit Open Source Software Kosten sparen?

Die Ergebnisse hängen stark vom Kalkulationsansatz stark ab. Zumindest bei den Lizenz- und Updatekosten sind Kostenvorteile offensichtlich. Der Betriebsaufwand in Technik, Dienstleistung und Personal kann Kostenvorteile aufweisen.

Ist es möglich, komplett auf Open Source umzustellen?

Das überwiegende Angebot an Open Source Software ist branchenneutral ausgerichtet. Insbesondere für die fachlichen Anwendungen der Sozialwirtschaft (Klientenverwaltung, Pflege- und Hilfemanagement, Dienstplanung) gibt es kaum Open-Source-Software-Angebote. Eine komplette Umstellung ist also nicht leistbar.

Darf ich Open Source Software in Ehrenamt und kommerziellen Bereich nutzen?

Dem kommerziellen Einsatz steht bei echter Open-Source-Software nichts entgegen, es existieren allerdings viele Lizenzvarianten. Prüfen Sie die Lizenzbedingungen der einzusetzenden Software (www.ifross.org). Open Source SoftwareLösungen können frei an Ehrenamtliche verteilt werden.

\section{Erhöhung der Liquidität im Alter durch Reverse Mortgage}

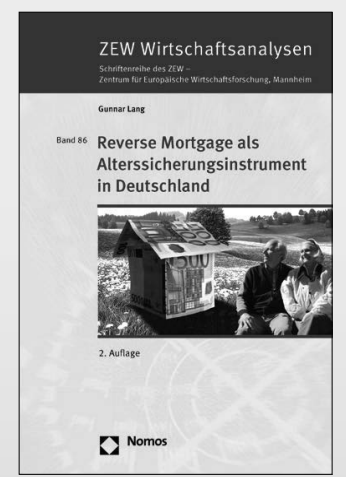

Reverse Mortgages sind Hypothekenkredite, die es selbst nutzenden Wohneigentümern ermöglichen, während der Vertragslaufzeit aus ihrem Wohneigentum ein monatliches Einkommen zur Liquiditätserhöhung im Alter

\author{
Reverse Mortgage als Alterssicherungs- \\ instrument in Deutschland \\ Von Gunnar Lang \\ 2. Auflage 2013, ca. 150 S., brosch., ca. 29,- $-€$ \\ ISBN 978-3-8329-6883-0 \\ (ZEW Wirtschaftsanalysen - \\ Schriftenreihe des ZEW, Bd. 86) \\ Erscheint ca. Oktober 2013
}

zu erwirtschaften. Der Autor untersucht die Eigenschaften von Reverse Mortgage und die Entwicklungen auf bestehenden Märkten, insbesondere hinsichtlich einer potenziellen Implementierung in Deutschland. 